\title{
Conservative Management of Separated Endodontic Instruments in the Root Canal - A Case Series
}

\author{
Omprakash Dulhani ${ }^{1}$, M. Mukherjee ${ }^{2}$ \\ ${ }^{1} \mathrm{BDS}, 354$ Field Hospital, C/o 56 APO, ${ }^{2} \mathrm{BDS}$, MDS (Perio), Army Dental Centre (R\&R), Delhi Cantt
}

Email for correspondence: dromprakashdulhani@gmail.com

\begin{abstract}
Root canal treatment is one of the most common procedures to be performed in all dental clinics nowadays. The popularity of the root canal treatment is attributed to the patients' awareness toward the dental treatment. Separation of the endodontic instrument is one of the common untoward incidents faced by the clinicians. Separated endodontic instrument can worsen the prognosis of root canal treatment by impeding the access beyond the broken instrument. This case series describes the conservative approach (bypass technique) to deal with broken instruments with minimum armamentaria. Furthermore, the bypassing technique does not carry much risk since it does not mandates significant removal of dentin structure.
\end{abstract}

Key words: Bypass, endodontic instruments, separated instruments

\section{INTRODUCTION}

The primary objectives of root canal treatment are proper cleaning, shaping, and threedimensional fluid-tight sealing of the root canal system. These objectives will not be achieved if an instrument gets separated in the canal. The success of root canal treatment declines markedly if the clinician fails to achieve the above said primary objectives. Evaluation of post-endodontic radiograph shows that $2-6 \%$ of the cases have separated instruments. ${ }^{[1]}$

Fracture often results from incorrect use or overuse of an endodontic instrument and seems to occur most commonly in the apical third of a root canal. Furthermore, fracture of rotary nickel titanium (NiTi) instruments may occur without warning, even with brand new instruments,

\section{Quick Response Code Article Info:}

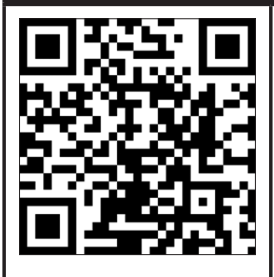

doi: 10.5866/2019.11.10052

Received: 08-04-2019

Revised: 08-05-2019

Accepted: 12-06-2019

Available Online: 01-07-2019, (www.

nacd.in) @ NAD, 2019 - All rights reserved whereas fracture of stainless steel files is preceded by instrument distortion serving as a warning of impending fracture. A disadvantage of NiTi alloy is its low ultimate tensile and yield strength compared with stainless steel, making it more susceptible to fracture at lower loads. ${ }^{[2]}$

Any error that proliferates microbial activity is likely to increase the risk of failure. Separated root canal instruments are one of the most troublesome incidents in endodontic therapy, especially if the tooth is non-vital and fragment cannot be removed. In the majority of cases, the procedural mishap does not directly compromise the prognosis, unless a concomitant infection is already present. Separated fragment of instrument in root canal may be indirectly responsible for an endodontic failure by limiting the access to the apical part of the canal, compromising disinfection and obturation, but is rarely directly responsible. Although several factors such as vitality of tooth, accessibility of tooth, position of fractured instrument in the canal, taper of the instrument, and type of alloy with which the instrument is made, have an impact on the prognosis, the presence of a periapical lesion served as the main prognostic factor for the successful treatment of such cases. ${ }^{[3]}$ 
The ideal solution of the problem is to remove the separated instrument without any complication. However, the retrieval of separated instruments is one of the most difficult operations in endodontic treatment, which is time consuming and requires skillful operation, advanced techniques, and professional equipment. In addition, there are considerable risks during the retrieval process, such as ledge formation, refracture of instruments, and perforation or vertical cracks due to over preparation of root canals. The previous studies have suggested that separated instruments in the lower segment of the root canal should be treated prudently. ${ }^{[4]}$

The ultimate goal of the management of separated endodontic instruments is not only to retrieve the fragment but also to preserve the integrity of the tooth. With the associated complications, bypassing a fragment located deep in the root canal or beyond the root canal curvature, if possible, may be the appropriate treatment option. To a great extent, this fulfills the objectives of root canal treatment: Proper cleaning and shaping of root canal treatment followed by good filling. Thus, bypassing the separated instrument has been categorized as a successful approach. ${ }^{[5]}$ In the present case report, three cases are reported where separated endodontic instruments were bypassed and treated conservatively.

\section{CASE REPORT 1}

A 35-year-old man reported with a chief complaint of toothache in upper right back tooth region for the past 2 days. Pain was incapacitating and continuous and the patient also gave a history of root canal treatment in the same tooth 2 years back in some village. He also had a similar episode a year back, which subsided on taking over-the-counter medication. On clinical examination, tooth number 15 was tender on percussion and on radiographic examination, root canal was not obturated; furthermore, a broken endodontic instrument (measuring approximately $2-3 \mathrm{~mm}$ ) was located in the apical third of the root canal [Figure 1]. Since separated instrument was present in the apical third and there was no periapical lesion associated with the tooth in consideration, bypass was planned.

On the first visit itself, a reentry into the chamber was made. Straight line access was established and two canals buccal and palatal were located, with the separated instrument being located in the buccal canal [Figure 1]. Bypassing was started with size $06 \mathrm{~K}$-file. Moreover, after a few attempts, bypass was achieved with $06 \mathrm{~K}$-file going past the broken instrument [Figure 2]. Working length was established using apex locator and confirmed radiographically. Crown-down technique was employed for cleaning and shaping of the canals. During the shaping of the canal, copious irrigation with $5.25 \%$ sodium hypochlorite and $\mathrm{N}$-saline was performed. Recapitulation was done with a size $10 \mathrm{~K}$-file between every instrument. After the completion of cleaning and shaping, calcium hydroxide was placed in the canals and the cavity was sealed with cotton pellet and temporary restoration. The patient was recalled after 2 weeks.

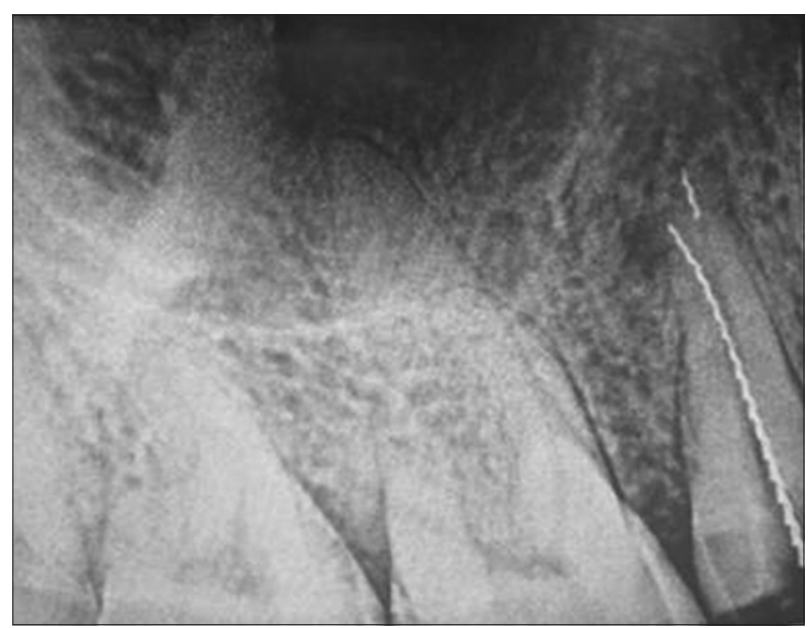

Figure 1: Intraoral periapical radiograph showing broken endodontic instrument measuring approximately $2-3 \mathrm{~mm}$, located in the apical third of the root canal

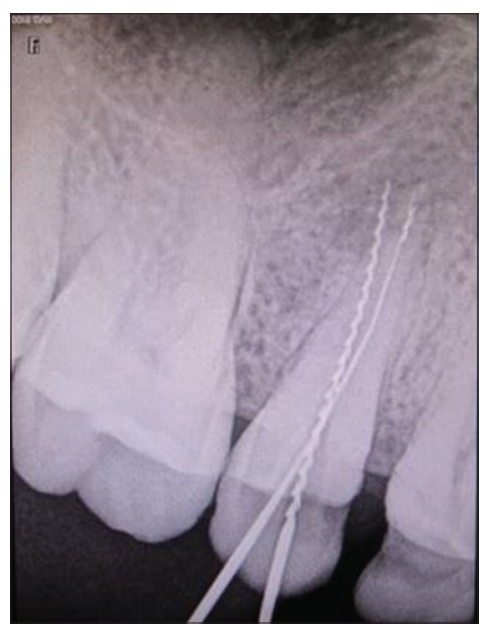

Figure 2: Bypass was achieved with $06 \mathrm{~K}$-file going past the broken instrument 
On the second visit, the patient being asymptomatic, calcium hydroxide dressing was removed by K-file and $\mathrm{N}$-saline irrigation. Cleaning and shaping was performed till apical preparation of 30 . Copious irrigation was done with $5.25 \%$ sodium hypochlorite and $\mathrm{N}$-saline. Canals were dried with paper points. Obturation was done with cold lateral condensation method [Figure 3]. Recall visits were uneventful and the patient is under observation.

\section{CASE REPORT 2}

A 41-year-old lady reported with the chief complaint of pain in the lower right back tooth region for the past 2 weeks; pain is continuous, severe in intensity, radiates to the head and ear, and relieves on medication. The patient also gave a history of root canal treatment 6 months back in the same region. On clinical examination, tooth number 46 had a huge restoration and was tender on percussion. Radiographic examination reveals root canal treated 46 with separated instrument in the middle-apical region of mesial root [Figure 4]. On the same visit, inferior alveolar nerve block was administered, intracoronal restoration removed, and reentry in the chamber was made. Access was modified to gain straight line access to the canals. The previous root canal fillings were removed from mesiobuccal, mesiolingual, and distal canals; furthermore, additional distobuccal canal was located by tracing the dentinal map.

Instrument in the mesiobuccal canal was present in the middle-apical third and beyond curvature; furthermore, no periapical lesion was associated with the mesial root. Hence, bypass was attempted.

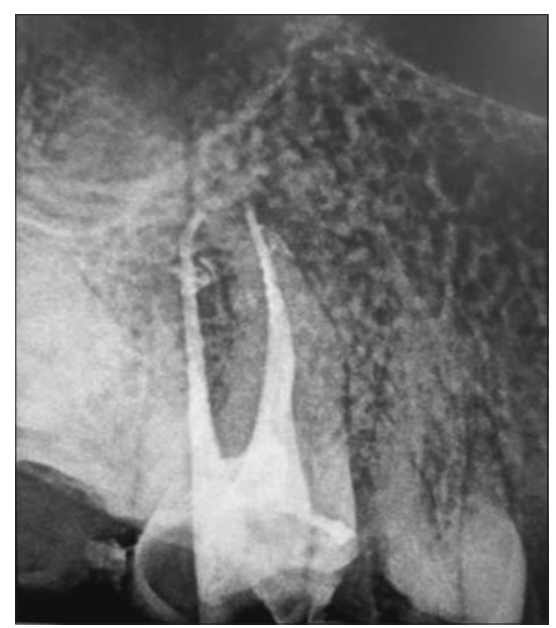

Figure 3: Post-obturation intraoral periapical radiograph showing complete obturation in 15
After a few initial attempts, the broken instrument was negotiated with 06 K-file [Figure 5]. Working length was established using apex locator and confirmed radiographically. Crown-down technique was employed for cleaning and shaping of the canals. During the shaping of the canals, copious irrigation with $5.25 \%$ sodium hypochlorite and $\mathrm{N}$-saline was performed. Recapitulation was done with a size 10 K-file between every instrument. Subsequently, mesiobuccal canal was instrumented till apical preparation of 30. Mesiolingual, distobuccal, and distolingual canals also instrumented till apical preparation of 30 .

After the completion of cleaning and shaping, calcium hydroxide was placed in the canals and the

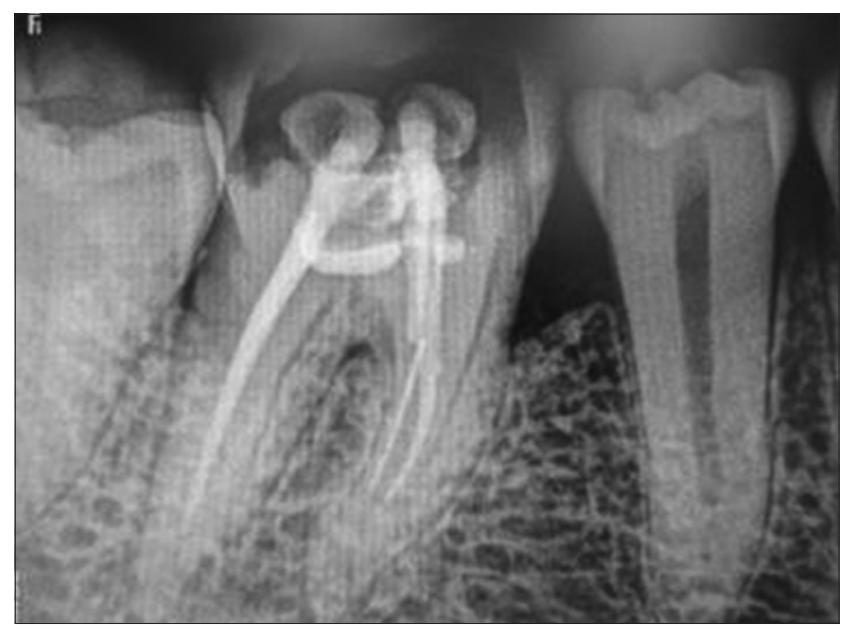

Figure 4: Intraoral periapical radiograph showing root canal treated 46 with separated instrument in the middle-apical region of mesial root

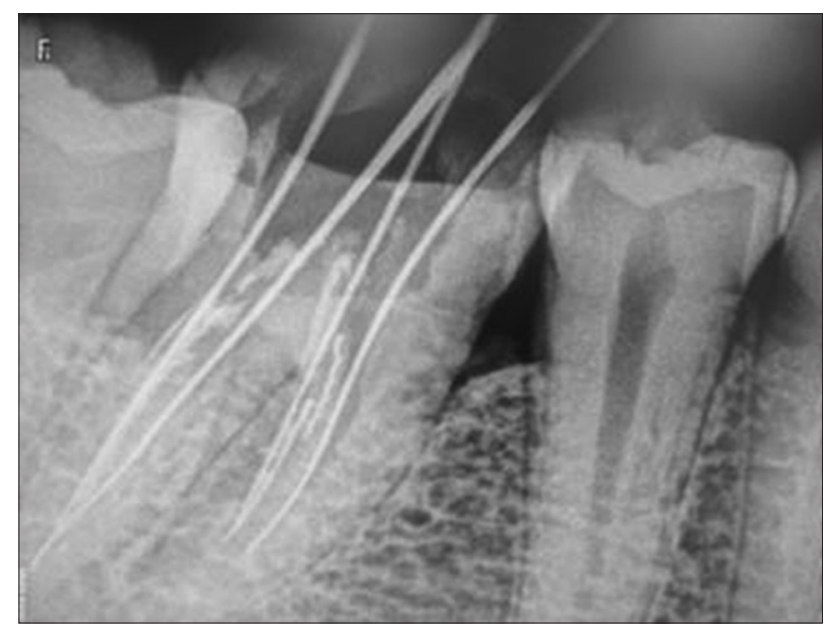

Figure 5: Bypass was achieved with $06 \mathrm{~K}$-file going past the broken instrument in the mesiobuccal canal of 46 
cavity was sealed with cotton pellet and temporary restoration. The patient was recalled after 3 weeks. On the second visit, the patient being asymptomatic, calcium hydroxide dressing was removed by K-file and $\mathrm{N}$-saline irrigation. Copious irrigation was done with $5.25 \%$ sodium hypochlorite and $\mathrm{N}$-saline. Canals were dried with paper points. Obturation was done with cold lateral condensation method [Figure 6]. Recall visits were uneventful and the patient is under observation.

\section{CASE REPORT 3}

A 32-year-old lady reported with a chief complaint of pain in the lower right back tooth region for the past 5 days. Pain is spontaneous, persists even after removal of the stimulus. On intraoral examination, tooth number 46 was found to have deep caries with suspected involvement of pulp. The tooth was tender on vertical percussion and vestibular tenderness was not present on palpation. A pre-operative radiograph was taken and tooth was found to have advanced caries with widening of periodontal ligament space in distal root. A provisional diagnosis of apical periodontitis irt 46 was made, by correlating the clinical and radiographic findings, and root canal treatment was planned. The patient was administered inferior alveolar nerve block and access opening was made, working length determined radiographically. Cleaning and shaping was started with hand files and followed by ProTaper rotary files, during the final stages of cleaning and shaping, $\mathrm{F} 1$ rotary file was separated in the mesiobuccal canal [Figure 7].

This mishap occurred while instrumentation of a tooth with vital pulp (without any periapical lesion) and during the final stages of cleaning and shaping. Hence, bypass was planned, and after a few initial attempts, broken instrument was negotiated with 06 K-file [Figure 8]. Moreover, subsequently, the mesiobuccal canal was prepared using 08, 10, 15, Sx, S1, S2 and F1 ProTaper hand files. Mesiolingual and distal canals were also prepared till F1, canals dried and closed dressing was given, and the patient recalled after 3 days. On the second visit, the patient was asymptomatic, copious irrigation was done with $5.25 \%$ sodium hypochlorite and $\mathrm{N}$-saline. Canals were dried with paper points. Obturation was done with cold lateral condensation method [Figure 9]. Recall visits were uneventful and the patient is under observation.

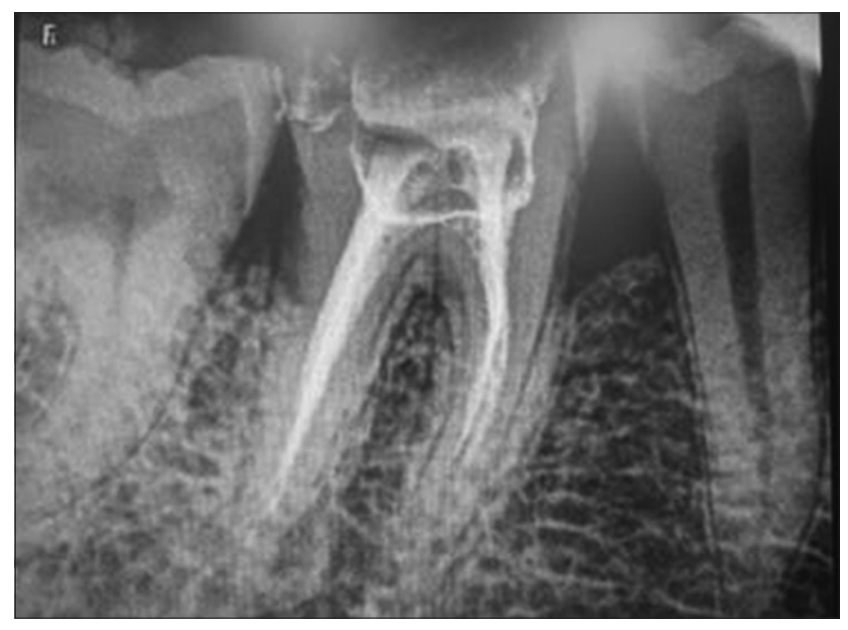

Figure 6: Post-obturation intraoral periapical radiograph showing complete obturation in 46

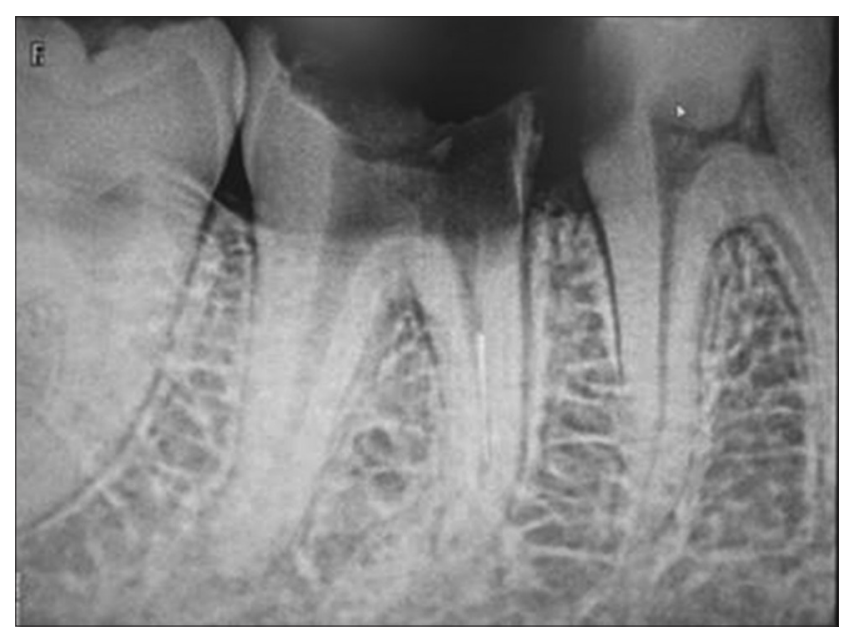

Figure 7: Intraoral periapical radiograph showing broken instrument in the mesiobuccal canal of 46

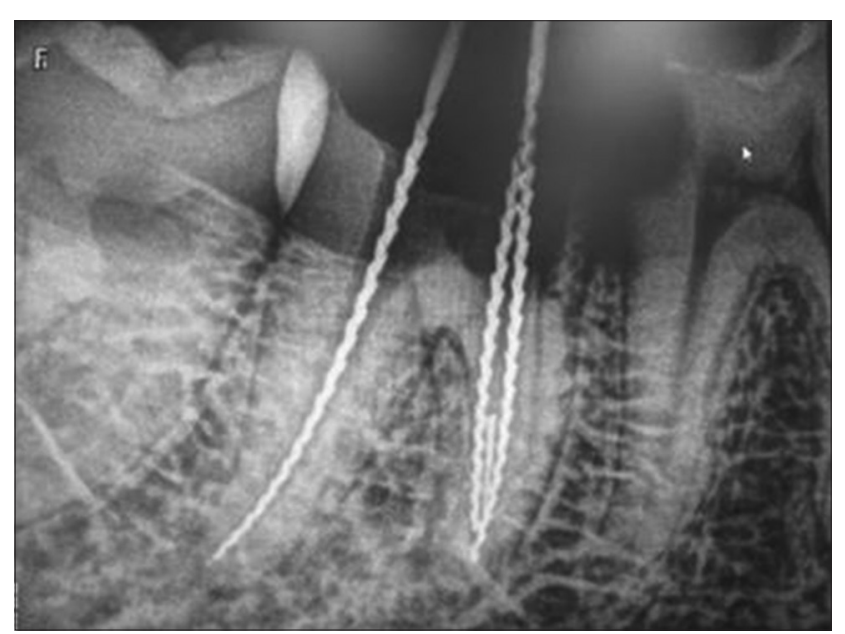

Figure 8: Bypass was achieved with $06 \mathrm{~K}$-file going past the broken instrument in the mesiobuccal canal of 46 


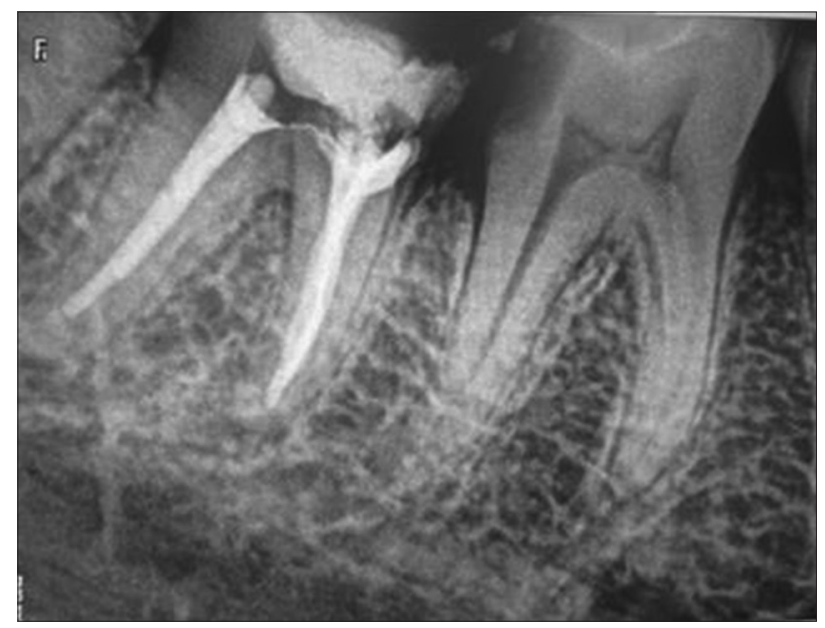

Figure 9: Post-obturation intraoral periapical radiograph showing complete obturation in 46

\section{DISCUSSION}

A review of literature reveals that the mean prevalence of retained fractured endodontic hand instruments (mostly stainless steel files) is in the range of $0.7-7.4 \%$. On the other hand, the mean clinical fracture frequency of rotary $\mathrm{NiTi}$ instruments is in the range of $0.4-3.7 \%$. Hence, based on the best available clinical evidence, the frequency of fracture of rotary NiTi instruments may actually be lower than that for stainless steel hand files. It is important to remember that reasons for fracture of rotary NiTi instruments are complex and multifactorial, one of the most important of which may be the operator's skill and experience. ${ }^{[2]}$

Interestingly, it was reported that $0.9 \%$ of previously unused $\mathrm{NiTi}$ instruments fracture during their first use perhaps due to misuse or a manufacturing defect. In addition, the probability of fracturing an instrument in the mesiobuccal canal of a maxillary molar was 3 times greater than the distobuccal canal. Similarly, the probability of fracturing a file in the mesiobuccal canal of a mandibular molar (known for their greater curvature) was greater than the mesiolingual canal. ${ }^{[6]}$

The patient must always be informed of the presence of the fragment and the proposed management. Decision on management should consider the following: (1) The constraints of the root canal accommodating the fragment, (2) the stage of root canal preparation at which the instrument separated, (3) the expertise of the clinician, (4) the armamentaria available, (5) the potential complications of the treatment approach adopted, (6) the strategic importance of the tooth involved, and (7) the presence/or absence of periapical pathosis. Clinical experience and understanding of these influencing factors as well as the ability to make a balanced decision are essential. The prognosis depends on the degree of contamination of canal at the moment of instrument separation..$^{[1,2,5]}$

The most ideal management of separated instruments is to prevent the occurrence in the first place. Adhering to proven concepts, integrating best strategies, and utilizing safe techniques during the root canal preparation procedures will virtually eliminate the broken instrument procedural accident. Prevention may also be greatly facilitated by thinking of negotiating and shaping instruments as disposable items. Simply discarding all instruments after the completion of each endodontic case will reduce breakage, lost clinical time, and upsets. However, on occasion an instrument will break and in spite of the best existing technologies and techniques, the broken file segment may not be able to be bypassed or retrieved. ${ }^{[7]}$

A management technique that does not require direct visibility to the fragment is "bypass," where a fine file is inserted between the fragment and root canal wall and thereby negotiating the canal to full working length and enable thorough instrumentation and obturation with the fragment remaining in situ. Incorporating the fragment in the root canal obturating material considerably improves the case prognosis. This technique does not require direct visibility to the fragment, i.e., it can be suitable even in cases when the fragment is located beyond a considerable root canal curvature. Furthermore, this method does not demand magnification aids strictly, as it is more dependent on tactile sensation of dentist. In this way, the root canal beyond the fractured instrument can be clean and the fractured instrument is incorporated in the root canal filling. In these cases, a good quality of obturation is mandatory so that the obturating material or sealer flows and seals the spaces between the flutes of separated file and canal wall. ${ }^{[1,3,8,9]}$

\section{CONCLUSION}

From the cases mentioned in this article, it can be concluded that bypassing the separated instrument is more conservative than retrieval. Recently, various specialized devices have been introduced specifically to retrieve fractured 
instruments. The problems associated with these devices include excessive removal of root canal dentin, ledging, perforation, limitation application in narrow and curved roots, and extrusion of the fractured portion through the apex. Straight line access is mandatory for successful removal of instruments, but conservation of tooth structure is paramount to the tooth's resistance to fracture.

This article concludes that once a fractured file is bypassed, the instrumentation of a root canal is best completed with hand file, to avoid further instruments fracture. With rotary files, the clinician's tactile sensation can be less than with hand files. In addition, when a rotary file contacts a metallic fragment while rotating, it becomes more fatigued. The friction between two metallic objects is expected to be greater than that between a rotary file and the dentine of root canal walls. Bypassing a fractured instrument fulfills, to a great extent, the objective of root canal treatment: Proper cleaning and shaping of root canal system followed by good obturation. ${ }^{[10,11]}$

\section{REFERENCES}

1. John P, Kumar MR, Jayasree S. Bypassing separated instruments in the root canal. IOSR J Dent Med Sci 2016;15:8-13.
2. Parashos P, Messer HH. Rotary NiTi instrument fracture and its consequences. J Endod 2006;32:1031-43.

3. Rambabu T. Management of fractured endodontic instruments in root canal: A review. J Sci Dent 2014;4:40-8.

4. Lin $\mathrm{C}, \mathrm{Xu}$ L, Chen YX, Liang Y, Chen XL, Lin Y, et al A statistical model for predicting the retrieval rate of separated instruments and clinical decision-making. J Dent Sci 2015;10:423-30.

5. Madarati AA, Hunter MJ, Dummer PM. Management of intracanal separated instruments. J Endod 2013;39:569-81.

6. McGuigan MB, Louca C, Duncan HF. Endodontic instrument fracture: Causes and prevention. Br Dent J 2013;214:341-8.

7. Kunhappan S, Kunhappan N, Patil S, Agrawal P. Retrieval of separated instrument with instrument removal system. J Int Clin Dent Res Organ 2012;4:21-4.

8. Shiyakov KK, Vasileva RI. Success for removing or bypassing instruments fractured beyond the root canal curve-45 clinical cases. J IMAB 2014;20:567-1.

9. Radeva E. Bypassing a broken instruments (clinical cases). Int J Sci Res 2017;6:227-9.

10. Madarati AA. Retrieval of multiple separated endodontic instruments using ultrasonic vibration: Case report J Taibah Univ Med Sci 2016;11:268-73

11. Monteiro JC, Kuga MC, Dantas AA, Jordão-Basso KC, Keine KC, Ruchaya PJ, et al A method for retrieving endodontic or atypical nonendodontic separated instruments from the root canal: A report of two cases. J Contemp Dent Pract 2014;15:770-4. 